\title{
Multi-State Grid Resource Availability Characterization
}

\author{
Brent Rood and Michael J. Lewis \\ Department. of Computer Science \\ State University of New York (SUNY) at Binghamton \\ Binghamton, NY, 13902 \\ \{brood1, mlewis\}@binghamton.edu
}

\begin{abstract}
The functional heterogeneity of non-dedicated computational grids will increase with the inclusion of resources from desktop grids, P2P systems, and even mobile grids. Machine failure characteristics, as well as individual and organizational policies for resource usage by the grid, will increasingly vary even more than they already do. Since grid applications also vary as to how well they tolerate the failure of the host on which they run, grid schedulers must begin to predict and consider how resources will transition between availability modes. Toward this goal, this paper introduces five availability states, and characterizes a Condor pool trace that uncovers when, how, and why its resources reside in, and transition between, these states. This characterization suggests resource categories that schedulers can use to make better mapping decisions. Simulations that characterize how a variety of jobs would run on the traced resources demonstrate this approach's potential for performance improvement. A simple predictor based on the previous day's behavior indicates that the states and categories are somewhat predictable, thereby supporting the potential usefulness of multi-state grid resource availability characterization. ${ }^{1}$
\end{abstract}

\section{INTRODUCTION}

The functionality, composition, utilization, and scale of computational grids continue to evolve. The largest grids and testbeds under centralized administrative control-including TeraGrid [26], EGEE [27], Open Science Grid (OSG) [28], and PlanetLab [29] - vary considerably in terms of the number of sites and the extent of the resources at each. Various peer-to-peer (P2P) [30] and Public Resource Computing (PRC) [25] systems allow users to connect and donate their individual machines, and to administer their systems themselves. We expect hybrids of these various grid models and middleware to emerge as well [31][32]. Future grids will contain dedicated high performance clusters, individual less-powerful machines, and even a variety of alternative devices such as PDAs, sensors, and instruments. They will run grid middleware, such as Condor [2] and Globus [33], that enables a wide range of policies for contributing resources to the grid.

This continued increase in functional heterogeneity will make grid scheduling - mapping applications onto available constituent grid resources - even more challenging, partly because different resources will exhibit varying unavailability characteristics. For example, laptops may typically be turned on and off more frequently, and join and leave the network

\footnotetext{
${ }^{1}$ This research is supported by NSF Career Award ACI-0133838 and NSF Award CNS-0454298.
}

more often. Individual workstation and desktop owners may turn their machines off at night, or shut down and restart to install new software more often than a server's system administrator might. Even the same kinds of resources will exhibit different availability characteristics. CS department research clusters may be readily available to the grid, whereas a small cluster from a physics department may not allow remote grid jobs to execute unless the cluster is otherwise idle. Site autonomy has long been recognized to be an important grid attribute [4]. In the same vein, even owners of individual resources will exhibit different usage patterns and implement different policies for how available they make their machines.

If grid schedulers do not account for these important differences, they will make poor mapping decisions that will undermine their effectiveness. On the other hand, schedulers that know when, why, and how the resources fail, can be much more effective, especially if this knowledge is coupled with information about application characteristics. For example, long-running jobs that do not implement checkpointing may require highly available and reliable host machines. Checkpointable applications that require heavyweight checkpoints may prefer resources that typically become unavailable to the grid "gracefully" (e.g. when a user returns to reclaim her machine), rather than when the machine itself goes down - in this case, the heavyweight checkpoint could be created on demand, rather than periodically, thereby avoiding unnecessary checkpointing overhead. This would be less important for applications that produce lightweight checkpoints. Easily replicable processes without side effects (e.g., those that are currently most appropriate for systems like BOINC [25]), might do well on the less powerful and more intermittently available resources, leaving the other machines for applications that really need them (at least under moderate to high contention for grid resources).

This paper describes our initial work toward building grid schedulers that consider unavailability characteristics of target resources. This goal requires progress in several directions. First, we must trace resources to uncover why and how they fail. As described in Section III, most availability traces focus primarily on when resources fail, and detail the patterns based purely on availability and unavailability. This paper identifies different types of unavailability, and analyzes their patterns.

We must also classify resources in a way that availability predictors can take advantage of, and design and build such predictors. Knowing that grid resources fail differently-and even predicting when and how they do-is important only to the extent that schedulers can exploit this information. 
Therefore, we must build schedulers that utilize both availability predictions - which are based on failure and unavailability characteristics - and application characteristics.

This paper takes initial steps toward failure-aware predictive grid scheduling. Section II identifies five resource availability states, dividing unavailable resources into separate categories based on why they are unavailable. This allows us to differentiate, for example, between resources that have failed, and resources whose CPU threshold has been exceeded We also describe the events that trigger resources to transition between availability states. We then classify applications by characteristics that could help schedulers, especially in terms of those machines' failure and unavailability characteristics.

Next, we describe traces that expose unavailability characteristics more explicitly than current traces. We identify the availability states that resources inhabit, and the transitions between them that resources make, over time. We report sample data from four months of traces on the University of Notre Dame's Condor pool; this data is not intended to necessarily exemplify exactly how all grid resources behave, but instead to illustrate how the trace methodology works, and to demonstrate the potential of our approach. This potential is illustrated by Section IV's classification of resources, Section V's simulation that identifies application failure rates on resources with different unavailability characteristics, and the potential performance increase when used with a simple predictor like the one we describe in Section VI.

To summarize, this paper's contributions include

- a new way of analyzing trace data, which better exposes resource failure characteristics,

- sample data from one such Condor-based trace,

- a new classification approach that enables effective categorization of resources into availability states

- results from a simulation that shows the potential benefit of failure-aware prediction and scheduling, and

- an initial simple predictor that demonstrates the utility of our trace and classification schemes.

We believe that these results make a strong case for studying how a grid's constituent resources become unavailable, for using that information to predict how individual resources may behave in the future, and for building that information into future grid schedulers, especially those that will operate in increasingly eclectic and functionally heterogeneous grids.

\section{AvaILABILITy ClassificAtion}

Resources in non-dedicated grids oscillate between being available and unavailable to the grid. When and how they do so depends on the failure characteristics of the machines, the policies of resource owners, the scheduling policies and mechanism of the grid middleware, and the characteristics of the grid's offered application load. This section identifies five availability states, and several application characteristics of applications that could influence their ability to tolerate resource faults. We focus our discussion and analysis on Condor, but the results translate to any system with Condor's basic properties of (i) non-dedicated distributed resource sharing, and (ii) a mechanism that allows resource owners to dictate when and how their machines are used by the grid.

\section{A. Condor}

Condor [1] harnesses idle resources from clusters, organizations, and even multi-institutional grid environments (via flocking and compatibility with Globus [33]) by integrating resource management, monitoring, scheduling, and job queuing components. Condor can automatically create process checkpoints for migration. Condor manages nondedicated resources, and allows individual owners to set their own policies for how and when they are utilized, as described below. Default policies dictate the behavior of resources in the absence of customized user policies [3], and attempt to minimize Condor's disturbance of local users and processes.

By default, Condor starts jobs only on resources that have been idle for 15 minutes, that are not running another Condor job, and whose local load is less than 30\%. Running jobs remain subject to Condor's policies. If the keyboard is touched or if CPU load from local processes exceeds $50 \%$ for 2 minutes, Condor halts the process but leaves it in memory, suspended (if its image size is less than $10 \mathrm{MB}$ ). Condor resumes suspended jobs after 5 minutes of idle time, and when local CPU load falls below $30 \%$. If a job is suspended for longer than 10 minutes or if its image exceeds $10 \mathrm{MB}$, Condor gives it 10 minutes to gracefully vacate, and then terminates it. Condor may also evict a job for a higher priority job, or if Condor itself is shut down.

Condor defaults dictate that applications whose checkpoints exceed $60 \mathrm{MB}$ checkpoint every 6 hours; those with larger images checkpoint every 12 hours. By default, Condor delivers checkpoints back to the machine that submits the job.

\section{B. Unavailability Types}

Condor's mechanism suggests a model that encompasses Figure 1's five availability states. By our definition, an Available machine is currently running with network connectivity, non-zero idle time, and a local CPU load of less than the CPU threshold. It may or may not be running a Condor job. A resource may transition to the User Present or CPU Threshold Exceeded state (i) if the keyboard or mouse is touched or (ii) if the local CPU load becomes too high, respectively. If the resource remains in either of these suspension states too long, if the job is evicted while running for any other reason, or if the machine is shut down, it transitions to the Job Eviction state. Finally, if a machine fails or becomes unreachable, it directly transitions to Unavailable.

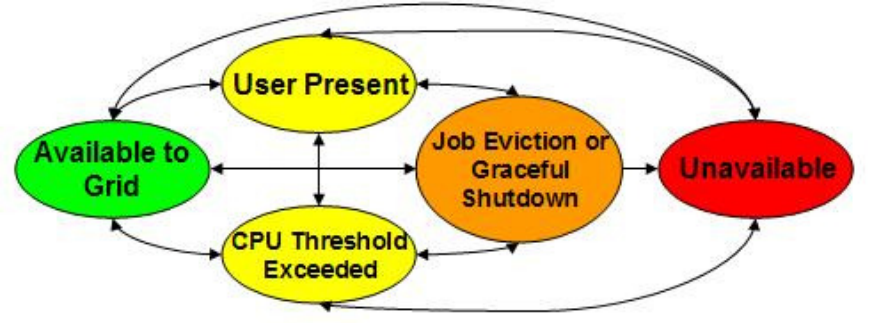

Figure 1: Multi-state Availability Model 
These states differentiate the types of unavailability. If a job is running or suspended, and enters the Job Eviction state, we call this a graceful transition to the Unavailable state; a transition directly to Unavailable is ungraceful.

This model is motivated by Condor's mechanism, but can reflect the policies that resource owners apply. For example, if an owner allows Condor jobs even when the user is present, the machine never enters the User Present state. Increasing local CPU threshold decreases the time spent in the CPU Threshold Exceeded state, assuming similar usage patterns. The model can also reflect the resource's job rank and suspension policy by showing when jobs are evicted directly without first being suspended.

\section{Grid Application Diversity}

Grid applications vary in their ability to tolerate faults. A checkpointable application need not be restarted from the beginning if its host resource transitions gracefully to Unavailable. Only Condor applications that run in the "standard universe" [3] are checkpointable. This requires relinking with condor compile, which does not allow jobs with multiple processes, interprocess communication, extensive network communication, file locks, multiple kernel level threads, files open for both reading and writing, or Java or PVM applications [3]. These restrictions demonstrate that only some grid jobs will be checkpointable. Another important factor is application runtime; grid applications may complete in a few seconds, or require many hours or even days [11]. Longer jobs will experience more faults, increasing the importance of their varied ability to deal with them.

Grid resources will have different characteristics in terms of how long they stay in each availability state, how often they transition between the states, and which states they transition to. Different applications will behave differently on different resources. If a standard universe job is suspended and then eventually gracefully evicted, it could checkpoint and resume on another machine. An ungraceful transition requires using the most recent periodic checkpoint. A job that is not checkpointable must restart from the beginning, even when gracefully evicted.

The point is that application characteristics, including checkpointability and expected runtime, can influence the effectiveness of scheduling those applications on resources that behave differently according to their transitions between the availability states identified in Section II.B. We explore this effect further in Section V.

\section{RELATED WORK}

Condor [2] is the best known cycle stealing system. The ability to utilize idle resources with minimal technical and administrative overhead has enabled long-term popularity and success. Resource management systems like Condor form the basis for our work. Other important related areas include resource state tracing, and prediction.

\section{A. Resource Availability Traces}

Several papers describe traces of workstation pools. Archarya et al. [7] take three 14 day traces at three university campuses, and analyze them for availability over time, and for the fraction of time a cluster of $k$ workstations is free. Bolosky et al. [10] trace more than 51,000 machines at Microsoft, and report the uptime distribution, the available machine count over time, and temporal correlations among uptime, CPU load per time of the week, and lifetime. Arpaci et al. [11] describe job submission by time of day and length of job, report machine availability according to time of day, and analyze the cost of process migration.

Other traces address host availability and CPU load in grid environments. Kondo et al. [5] [6] analyze grid traces from an application perspective, by running fixed length CPU bound tasks. The authors analyze availability and unavailability durations during business and non-business hours, in terms of time and number of operations [5]. They also show the cumulative distribution of availability intervals versus time and number of operations [6]. The authors derive expected task failure rates based but state that the traces are unable to uncover the specific causes of unavailability (e.g. user activity vs. host failure). Our traces borrow from this general approach.

Ryu and Hollingsworth [9] describe fine grained cycle stealing (FGCS) by examining a NOW trace [11] and a Condor trace for non-idle time and its causes (local CPU load exceeding a threshold, local user presence, and the time the grid must wait after a user has left before utilizing the resource). Anderson and Fedak [12] analyze BOINC hosts for computational power, disk space, network throughput, and number of hosts available over time (in terms of churn, lifetime, and arrival history). They also briefly study onfraction, connected-fraction, active-fraction, and CPU efficiency. Finally, Chun and Vahdat [13] report PlanetLab all pairs ping data, detailing resource availability distribution and mean time to failure.

Unlike the work described above, our traces are designed to uncover the causes of unavailability. We analyze trace data and classify machines into our own availability states. We analyze how individual resources in the various states behave over time (by both day and hour). Our less extensive traces are intended primarily to motivate our approach to availability awareness and prediction in grid scheduling.

\section{B. Availability and Load Prediction}

The most notable prediction system for grids and networked computers is Network Weather Service (NWS) [23]. NWS uses a large set of linear models for host load prediction, and combines them in a mixture-of-experts approach that chooses the best performing predictor. The RPS toolkit [24] uses a set of linear host load predictors. Availability prediction based on different causes of unavailability is currently outside the scope of these projects.

In availability prediction, Ren et al. [14][15] use empirical host CPU utilization and resource contention traces to develop a multi-state model for resource availability, and analyze availability and unavailability durations by hour of day. Their multi-state availability model includes five states, three of which are based on the CPU load level (which resides in one of three zones). The two other states indicate memory thrashing and resource unavailability. This model neither 
captures user presence nor allows for differentiation between graceful and ungraceful process eviction. For prediction, the authors use the previous $N$ days of a resource's history to produce a Markov chain for state transition predictions.

Pietrobon and Orlando [16] use regressive analysis of past job executions to predict whether a job will succeed. Mickens and Noble [17][18][19] use variations and combinations of saturating counters and linear predictors, including a hybrid approach much like NWS's mixture-of-experts, to predict the likelihood of a host being available for various look ahead periods. Nurmi et al. [8] model machine availability using Condor traces and an Internet host availability dataset, attempt to fit Weibull, hyper-exponential and Pareto models to the availability duration data, and evaluate them in terms of "goodness-of-fit" tests. They then provide confidence interval predictions for availability duration based on model-fitting [13]. Several machine learning techniques use categorical time-series data to predict rare target events by mining event sets that frequently precede them [20][21][22].

Our approach differs primarily in that we attempt to predict not just the availability of a resource, but also the way in which a resource is likely to become unavailable according to our multi-state availability model. As discussed later, the cause of unavailability can be exploited for performance gain.

\section{TRACE ANALYSIS}

We accessed, organized, and analyzed data in a four month Condor resource pool trace at the University of Notre Dame ${ }^{2}$ in early 2007. The trace consists of time-stamped CPU load (as a percentage) and idle time (in seconds). Condor records these measurements approximately every 16 minutes, and makes them available via the condor_status command. Idle times of zero imply user presence, and the absence of data indicates that the machine was down.

The data recorded by Condor precludes determining whether the Job Eviction or Graceful Shutdown state was entered, because intentional shutdown and machine failure appear the same in the data. Since unavailability is relatively uncommon and irregular, we conservatively assume that all transitions to unavailability are ungraceful. Also, we only consider a machine to be in the user state after the user has been present for 5 minutes; this policy filters out short unavailability intervals that lead to application suspension, not gracefully eviction. We consider a machine to be in the CPU Threshold Exceeded state if its local (i.e. non-Condor) load is above $50 \%$. Otherwise, a machine that is online with no user present and CPU load below $50 \%$ is considered Available. This includes machines currently running Condor jobs, which are clearly available for use by the grid. On SMP machines, we follow Condor's approach of treating each processor as a separate resource.

We processed and analyzed the trace data to categorize resources according to our states. Our goal is to identify trends that enable multi-state availability prediction and scheduling.

\footnotetext{
${ }^{2}$ We thank Douglas Thain for an account at Notre Dame, and permission to use the trace data.
}

\section{A. Condor Pool Characteristics}

This section examines the pool of machines as a whole, to enable conclusions about their aggregate behavior.

1) Pool State over Time: Figure 2 depicts the number of machines in each availability state over time. Gaps in the data indicate brief intervals between the four months' data. The data shows a diurnal pattern; availability peaks at night, and recesses during the day, but rarely below 300 available machines. This indicates workday usage patterns and a policy of leaving machines turned on overnight, exhibited by the diurnal User Present pattern. The number of machines that occupy the CPU Threshold Exceeded state is less reflective of daily patterns. Local load appears to be less predictable than user presence, and the number of unavailable machines also does not exhibit obvious patterns.
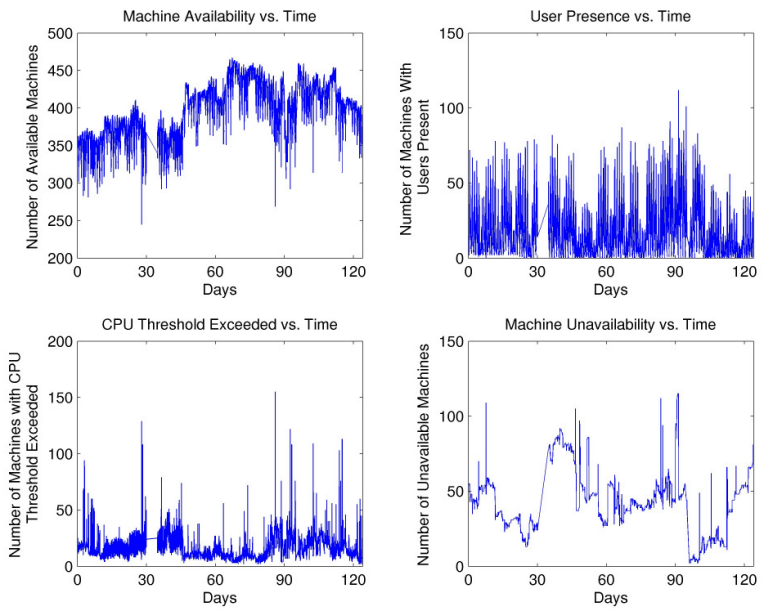

Figure 2: Machine State over Time

Figure 3 depicts the overall percentage of time spent by the collective pool of resources in each availability state. The Available state encompasses $84.1 \%$ of the total time, followed by Unavailable (9\%), and transition states User Present and CPU Threshold Exceeded (3.3\% and 3.4\% respectively).

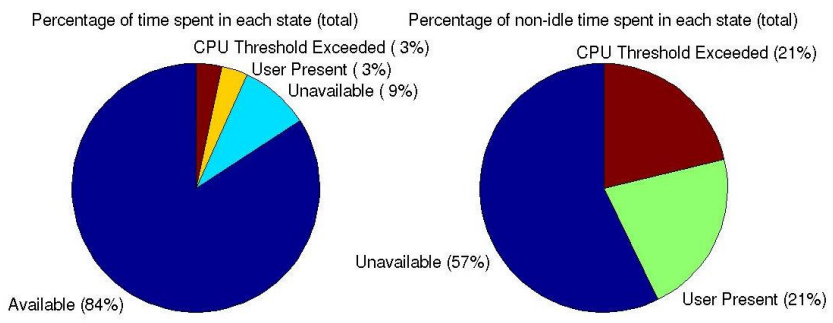

Figure 3: Total Time Spent in Each Availability State

2) Daily and Hourly Transitions and Durations: We also examine how often resources transition between states, and how long they reside in those states. We report the data as a function of both the day of the week and the hour of the day.

State transitions can affect the execution of an application because they can trigger application suspension, lead to 
checkpointing and migration, or even require restart. Figure 4 shows the number of transitions to each state by hour of day.
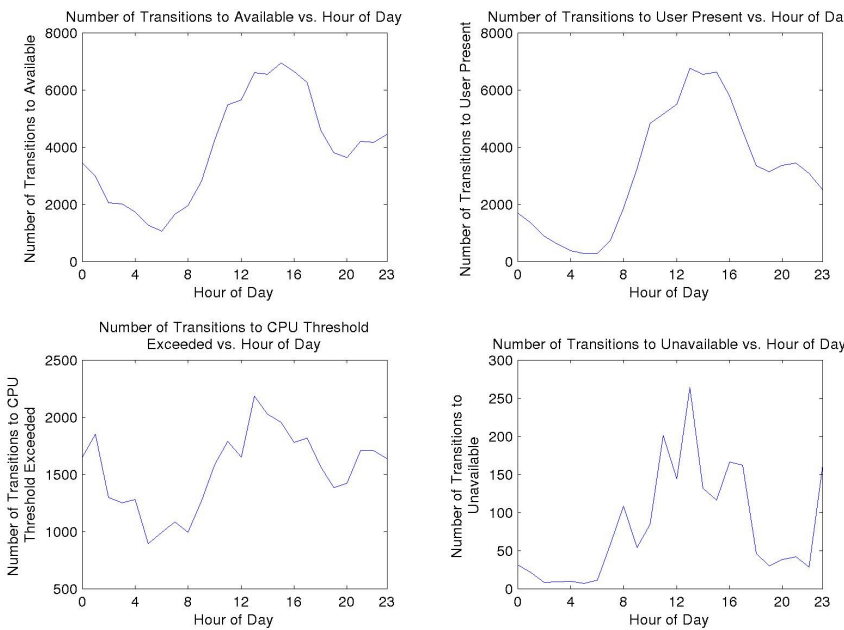

Figure 4: Number of Transitions versus Hour of the Day

Again, the numbers of transitions to both the Available and User Present states show clear diurnal patterns. Users return to their machines most often at around $3 \mathrm{pm}$, and most seldom at $5 \mathrm{am}$. Machines also frequently become available at Midnight and 10AM. Transitions to CPU Threshold Exceeded and Unavailable states also seem to exhibit similar (but slightly less regular) patterns.
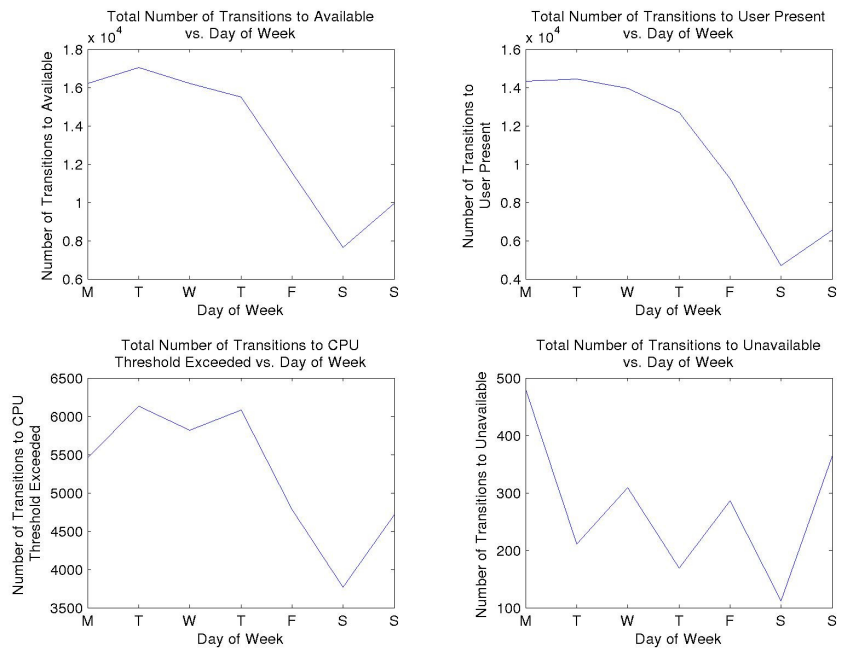

Figure 5: Number of Transitions versus Day of the Week

Figure 5 reports the daily pattern of machine transitions among states. Transitions to Available, User Present and CPU Threshold Exceeded fall from a high on Monday and Tuesday to a low on Saturday. Unavailability transitions peak on Sunday and Monday.

Next, we investigate state duration and its patterns. Figure 6 plots the average duration that resources remain in each state, beginning at each hour of the day. Expectedly, the average duration of availability is at its lowest in the middle of the day, since this is when users are present. Interestingly, CPU Threshold Exceeded and Unavailable durations both reach their minimum at mid-day, meaning that whereas machines are not typically completely free, they also are not running CPU intensive non-Condor jobs.
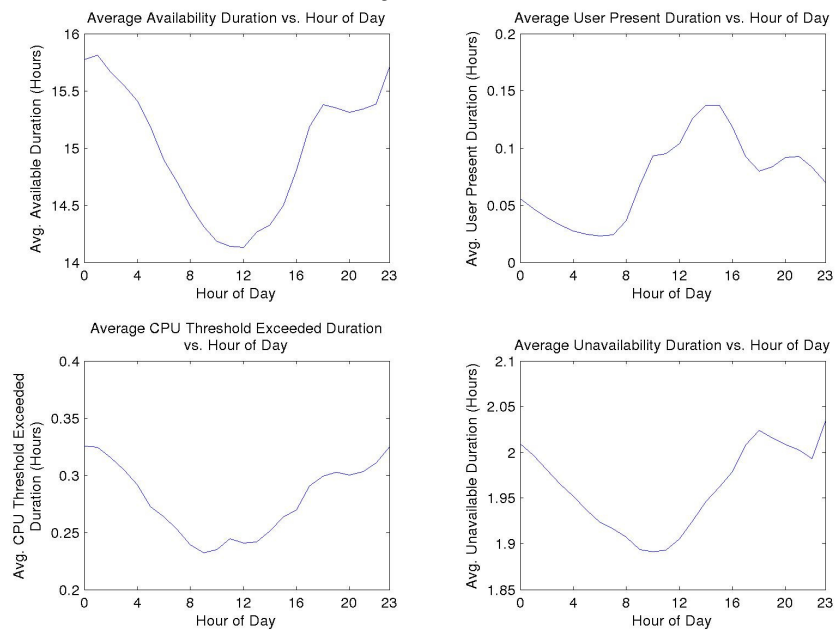

Figure 6: State Duration versus Hour of the Day

Unavailability duration is expectedly higher at night, when users abandon their machines until morning. The duration of the CPU Threshold Exceeded state is at its lowest during the day, most likely due to short bursts of daytime activity and longer, non-Condor jobs being run overnight.
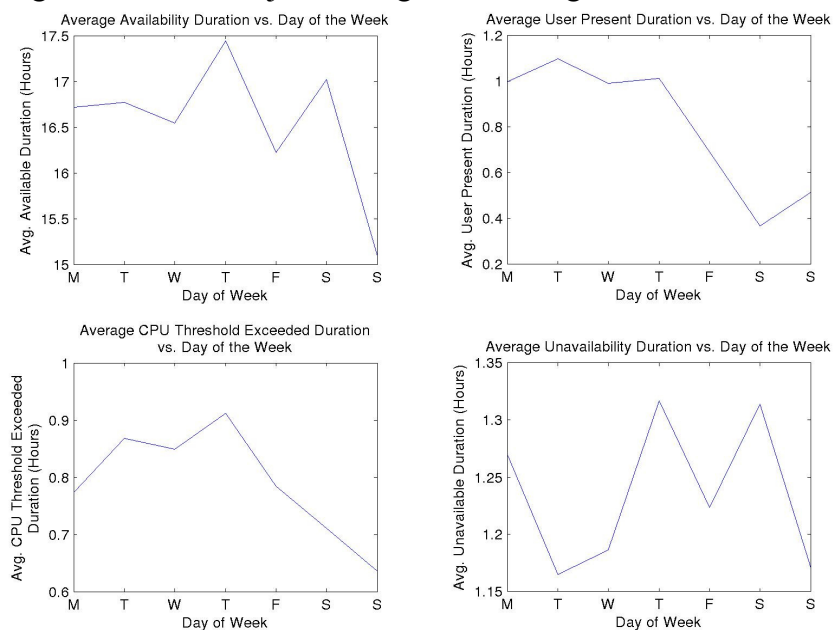

Figure 7: State Duration versus Day of the Week

Finally, Figure 7 examines the weekly behavior of the duration that resources reside in each state. The average length of time spent in the Available, User Present, and CPU Threshold Exceeded states follow similar patterns, although Available duration seems to be less consistent. The Unavailable state duration exhibits no obvious pattern.

\section{B. Individual Machine Characteristics}

This section examines the characteristics of individual resources in the pool. Figure 8 and Figure 9 plot cumulative machine counts such that an $(\mathrm{x}, \mathrm{y})$ point in the graph indicates that $y$ machines exhibit $x$ or fewer (i) average hours per day in a state (Figure 8) or (ii) number of transitions per day to a state (Figure 9). The first aspect we explore is how long, on average, each node remains in each state. 

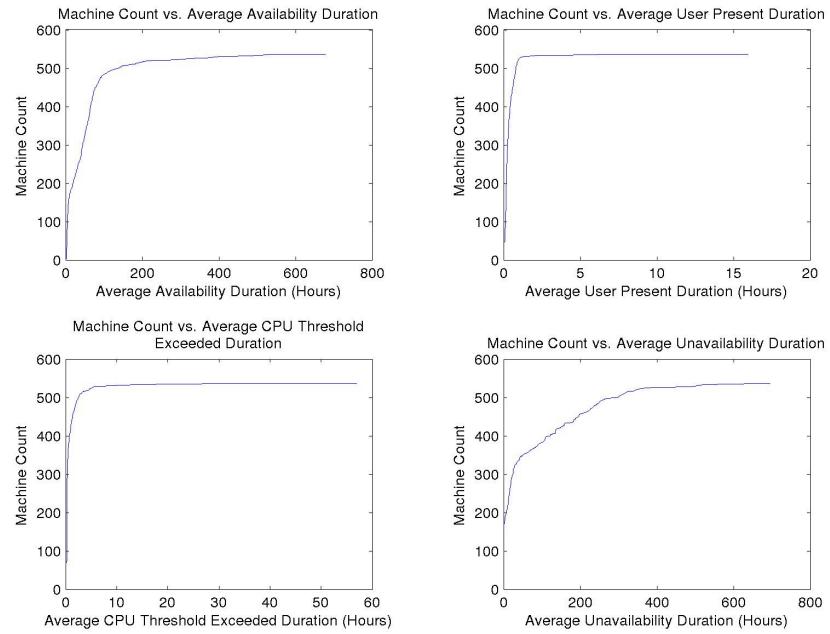

Figure 8: Machine Count versus State Duration

Figure 8 shows the distribution according to the average time spent in each of the four states. For Availability, the data suggests three distinct categories: 144 resources average 7.5 hours or less, 328 average between 7.5 and 89 hours, and the top 65 resources average more than 89 hours.

For User Present, 426 resources average less than 30 minutes of user presence; of those, 44 have no user activity. The remaining 67 resources have longer (over 30 minutes) average durations of user presence. For CPU Threshold Exceeded, 68 have no local load occurrences, 172 resources have short bursts of 17 minutes or less, 289 resources have longer average usage duration but less than 5.5 hours, and the remaining 9 resources remain in their high usage state over 5.5 hours on average. Average time in the Unavailable state shows that 311 resources have durations less than 25 hours; 128 have no unavailability. The top 38 resources have unavailability durations exceeding 284 hours (mostly offline).

Figure 9 examines the average number of resource transitions to each state per resource per day. $73.5 \%$ of resources transition to Available fewer than twice per day. The top $2 \%$ transition over 7 times on average. As reported earlier, 44 machines $(8 \%)$ have no user activity. On the other hand, 378 machines (70\%) infrequently experience local users becoming present (less than 2 transitions to User Present per day). Users frequently come and go on the remaining 115 resources $(21.4 \%)$, with some of these experiencing up to 10 transitions to User Present on average per day.

CPU Threshold Exceeded transitions are bipolar with 460 (85.6\%) machines having 1 or fewer transitions to CPU Threshold Exceeded per day (68 of those 400 have none). The remaining machines average more than 1 transition and peak at 5 transitions each day. Finally, unavailability is relatively uncommon with 128 resources $(23.8 \%)$ having no unavailability, $97(18 \%)$ transitioning to Unavailable only once, and only the top 40 machines (7.4\%) having ten transitions or more.

\section{Machine Classification}

This section classifies resources by considering multiple state characteristics simultaneously. Our approach organizes resources based on which type of applications they would be most useful in executing. In particular, the average availability duration dictates whether the application is likely to have complete, and how a machine transitions to Unavailable (gracefully or ungracefully) determines whether the job can checkpoint before migrating.

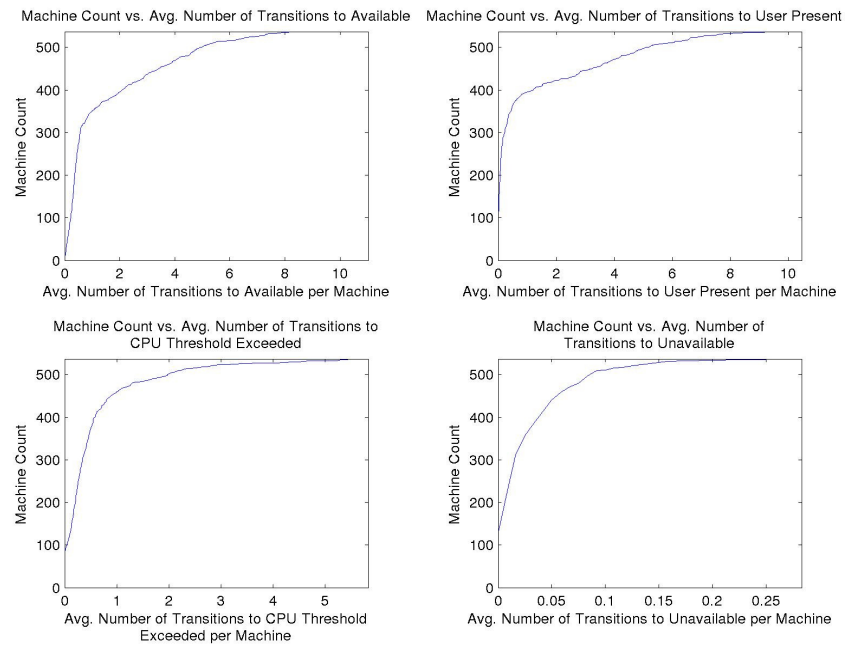

Figure 9: Machine Count vs. Average Number of Transitions Per Day

We classify the machines based on average availability duration and the number of graceful and ungraceful transitions to Unavailable per day. Table 1 reports the number of machines that fit these categories. The thresholds for the Availability categories are 65.5 (High), 39.7 (Medium High), and 6.5 (Medium Low) hours. We considered more than 1.6 graceful transitions to Unavailable, per day, to be High, and two or more ungraceful transitions in the months to be High.

TABLE 1

\begin{tabular}{|r|c|c|c|c|}
\cline { 2 - 5 } \multicolumn{1}{c|}{} & \multicolumn{4}{c|}{ Average \# of Graceful Transitions } \\
\multicolumn{1}{c|}{ High } & \multicolumn{3}{c|}{ Low } \\
\hline Average Availability & \multicolumn{4}{c|}{ Ungraceful Transitions } \\
Duration & High & Low & High & Low \\
\hline High & 0 & 0 & 44 & 90 \\
\hline Medium High & 0 & 0 & 30 & 104 \\
\hline Medium Low & 22 & 18 & 47 & 47 \\
\hline Low & 98 & 32 & 3 & 2 \\
\hline
\end{tabular}

Roughly $36 \%$ of machines have Medium High or High availability durations with both a low number of graceful and ungraceful transitions, making them highly available and unlikely to experience any kind of job eviction. In contrast, about $24 \%$ of resources exhibit low availability and a high number of graceful transitions; about $75 \%$ of these have high ungraceful transitions. The low availability group would be most appropriate for shorter running checkpointable applications. The last significant category (approximately $8.7 \%$ ) has Medium Low availability and few graceful and ungraceful transitions. This group lends itself to shorter running applications with or without the ability to checkpoint.

\section{PREDICTION MOTIVATION}

This section demonstrates the implications of Table 1's resource classification distribution. To test how applications 
would succeed or fail on resources in the different classes, we simulated a series of 100 jobs with runtimes equally distributed from approximately 5 minutes to 12 hours (on a machine running at 2500 MIPS). The simulation used each resource's local load at each point in the trace described in Section IV, along with the MIPS score that Condor calculates. Each job was begun at a random available point in each resource's trace and then the execution was simulated. This was done 1000 times for each job duration on each resource.

We include results only for the resources classified as (i) low availability with high graceful and ungraceful transitions (18\% of the machines), (ii) medium-low availability with low graceful transitions, but high ungraceful transitions (8.7\%), (iii) medium-high availability with few graceful and ungraceful transitions (19.3\%), and (iv) high availability, also with few graceful and ungraceful transitions (16.7\%). These classes represent a broad spectrum of the classification scheme and $62.7 \%$ of the total resources. They appear shaded in Table 1 , and are labeled (i) LA-HG-HUG, (ii) MLA-LG-HUG, (iii) MHA-LG-LUG, and (iv) HA-LG-LUG in Figure 10.

Figure 10 shows that graceful failure rates climb more rapidly for less available resources. The low availability resources also have a much higher ungraceful failure rate versus the high and medium high availability resources, which have a very low ungraceful failure rate. Interestingly, the resources with medium low availability have a larger ungraceful failure rate than the low availability machines.
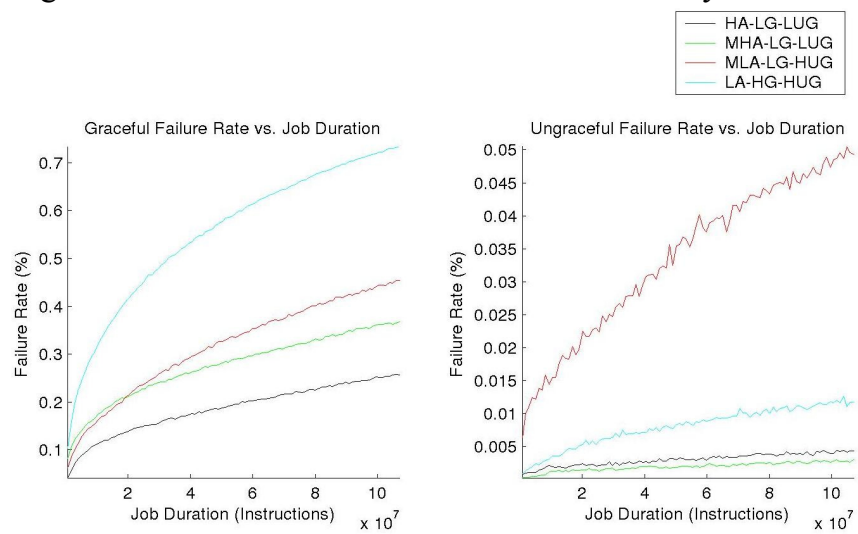

Figure 10: Failure Rate vs. Job Duration

These results are important because of the diversity of grid applications, as described in Section II.C. Checkpointable applications can tolerate graceful evictions by checkpointing on demand and resuming elsewhere, without duplicating work However, these same applications are more sensitive to ungraceful evictions; the amount of lost work due to an ungraceful transition to the Unavailable state depends on the checkpoint period. On the other hand, applications that do not checkpoint are equally vulnerable to graceful or ungraceful transitions; both require a restart. Therefore, resources in different classes should host different types of applications.

Intuitively, to make the best matches under high demand for resources, a scheduler should match the job duration with the expected availability duration of the machine; machines that are typically available for longer durations should be reserved, in general, for longer running jobs. And non- checkpointable jobs should run on resources that are not likely to transition to Unavailable. Finally, machines with high rates of ungraceful transition to Unavailability should perhaps be reserved for highly replicable jobs.

A second simulation demonstrates the importance of the classes. We created 1000 jobs of random length (equally distributed between 5 minutes and 12 hours), half of which were checkpointable with a period of six hours. We tested a scheduler that mapped jobs to resources randomly, and one that prioritized classes according to Table 1 . The simulator iterates through the trace, injecting an identical random job set and scheduling it with each scheduler. Job execution considers each resource's dynamic local load and MIPS score. When a resource that is executing a job becomes unavailable in the trace, the job is rescheduled. If the job is checkpointable and the transition is graceful, the job takes a fresh checkpoint and migrates. An ungraceful transition to unavailability requires restarting from the last successful periodic checkpoint. Noncheckpointable jobs must always restart from their beginning.

The classifier used months one and two to classify the machines; schedulers ran in months three and four. Each subsequent month was simulated three times and the average values were taken for that month. Table 2 reports the sum of those averages across both months. Jobs meet more evictions and therefore take an average of $31.6 \%$ longer when the scheduler ignores the unavailability characteristics.

TABLE 2

\begin{tabular}{|l|r|r|r|}
\hline & \multicolumn{1}{|c|}{$\begin{array}{c}\text { Random } \\
\text { Scheduler }\end{array}$} & $\begin{array}{c}\text { Classifier } \\
\text { Scheduler }\end{array}$ & Improvement \\
\hline Completed Jobs & 1883.2 & 1936.0 & $2.27 \%$ \\
\hline $\begin{array}{l}\text { Gracefully } \\
\text { Evicted Jobs }\end{array}$ & 1244.0 & 492.0 & $60.45 \%$ \\
\hline $\begin{array}{l}\text { Ungracefully } \\
\text { Evicted Jobs }\end{array}$ & 28.6 & 14.3 & $50.0 \%$ \\
\hline $\begin{array}{l}\text { Avg. Execution } \\
\text { time (secs.) }\end{array}$ & 71209.0 & 48656.0 & $31.6 \%$ \\
\hline
\end{tabular}

\section{PREDICTION}

The improvement reported above requires a scheduler that knows a resource's unavailability characteristics. To determine the feasibility of predicting Availability states, we developed a simple online predictor, and a simulator to test its effectiveness. Previous prediction work [14][15] has indicated that predictions based on the previous weekday or weekend have had moderate success. Section IV.A.2) also shows both daily and weekly patterns that justify such a prediction strategy. Therefore, we have implemented a predictor that uses the previous weekday or weekend day in determining the likelihood of transitioning to each state. The predictor takes as input a time interval, and uses data from the previous weekday or weekend day to output the predicted likelihoods of finishing the interval in the Available state, and of transitioning to User Present, CPU Threshold Exceeded, and Unavailable states.

Figure 11 plots the accuracy of each prediction type given the duration of the interval requested. Accuracy is the percentage of time that the predictor accurately forecasts that a particular state is entered next,. Approximately $20 \%-30 \%$ of 
the time that a machine was predicted to exit gracefully into the CPU Threshold Exceeded state, it actually did. Predictions for completion of the interval in the Available state follow a linear decrease in accuracy as the duration of the interval increases. Predictions for transitioning to the User Present state become dramatically more accurate as the prediction interval increases. Finally, predictions that indicate that Unavailable is the most likely next state have virtually no accuracy. Whereas this doesn't indicate that unavailability is completely unpredictable, it does demonstrate that the previous day is not a good indicator for this state.

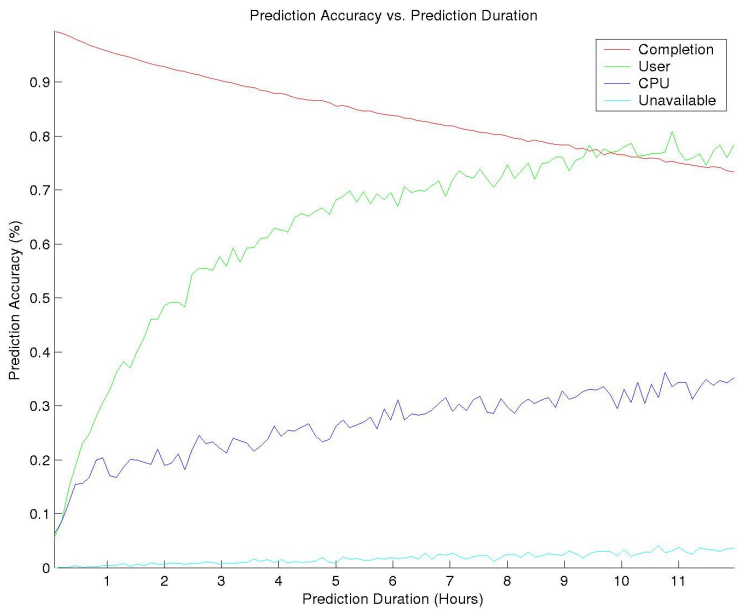

Figure 11: Prediction Accuracy vs. Prediction Duration

\section{SUMMARY}

This paper introduces five resource availability states, which capture why and how (not just when) resources become unavailable over time. This approach suggests a categorization of resources that can distinguish between graceful and ungraceful transitions to unavailability. This categorization is potentially useful for grid schedulers that also have useful information about an application's characteristics. In particular, the checkpointability and expected runtime of a job-in concert with predicted resource availability, and organized according to the strategies that we advocate in this paper-can suggest better mappings. We describe how one month of data from a university Condor pool slots resources into states, over time, and how these resources can then be classified according to trends and behavior. We demonstrate by simulation that mapping jobs of different lengths onto resources in these different categories has a significant effect on whether they can run to completion. Finally, we implement a very simple availability state predictor that demonstrates some potential for practical online prediction of future resource availability behavior, in terms of the availability states we introduce.

\section{REFERENCES}

[1] (2007) Condor;. http://www.cs.wisc.edu/condor/description.html

[2] M. Litzkow, M. Livny, and M. Mutka, "Condor - A Hunter of Idle Workstations," ICDCS '88, pp. 104-111.

[3] (2007) Condor Version 6.9.2 Manual. [Online]. Available: http://www.cs.wisc.edu/condor/manual/v6.9/

[4] M. Lewis and A. Grimshaw, "The Core Legion Object Model," HPDC '96, Aug. 1996, pp. 551-561.
[5] D. Kondo, G. Fedak, F. Cappello, A. A. Chien, and H. Casanova, "Resource Availability in Enterprise Desktop Grids," Dept. of Computer Science, INRIA, Technical Report 00000994, Jan. 2006.

[6] D. Kondo, M. Taufer, C.. Brooks III, H. Casanova and A. Chien, "Characterizing and Evaluating Desktop Grids: An Empirical Study," IPDPS'04, 2004, p. 26b.

[7] A. Acharya, G. Edjlali and J. Saltz, "The Utility of Exploiting Idle Workstations for Parallel Computation," SIGMETRICS '97, pp. 225-34.

[8] D. Nurmi, J. Brevik, and R. Wolski, "Modeling machine availability in enterprise and wide-area distributed computing environments," in, 2005, p. 432-441.

[9] K. Ryu and J. Hollingsworth, "Unobtrusiveness and Efficiency in Idle Cycle Stealing for PC Grids," in Proc. IPDPS'04, 2004, p. 62a.

[10] W. Bolosky, J. Douceur, D. Ely and M. Theimer, "Feasibility of a serverless distributed file system deployed on an existing set of desktop PCs," SIGMETRICS'00, 2000, pp. 34-43.

[11] R. Arpaci, A. Dusseau, A. Vahdat, L. Liu, T. Anderson and D. Patterson, "The interaction of parallel and sequential workloads on a network of workstations," SIGMETRICS'95, 1995, p.267-278.

[12] D. Anderson and G. Fedak, "The Computational and Storage Potential of Volunteer Computing," CCGRID'06, 2006, p. 73-80.

[13] B. Chun and A. Vahdat, "Workload and failure characterization on a large-scale federated testbed," Intel Research Berkeley, Tech. Rep. IRB-TR-03-040, Nov. 2003.

[14] X. Ren, S. Lee, R. Eigenmann and S. Bagchi, "Resource. Failure Prediction in Fine-Grained Cycle Sharing System," HPDC '06, 2006.

[15] X. Ren and R. Eigenmann, "Empirical Studies on the Behavior of Resource Availability in Fine-Grained Cycle Sharing Systems," in Proc. ICPP'06, 2006, p. 3-11.

[16] V. Pietrobon and S. Orlando, "Performance Fault Prediction Models," Dept. of Computer Science, University of Venice, Technical Report CS-2004-3, May 2004.

[17] J. Mickens and B. Noble, "Exploiting availability prediction in distributed systems," in Proc. NSDI'06, 2006, p. 73-86.

[18] J. Mickens and B. Noble, "Predicting node availability in peer-to-peer networks," in SIGMETRICS'05 poster, 2005.

[19] J. Mickens and B. Noble, "Improving distributed system performance using machine availability prediction," SIGMETRICS'06 Performance Evaluation Review, Sep. 2006, v.34 n.2.

[20] R. Vilalta and S. Ma, "Predicting Rare Events in Temporal Domains," in Proc. ICDM'02, 2002, p. 474.

[21] G. Weiss and H. Hirsh, "Learning to Predict Rare Events in Categorical Time-Series Data," in Proc. ICML'98 Workshop on "Predicting the Future: AI Approaches to Time-Series Problems", 1998, p. 83-90.

[22] R. Sahoo, A. Oliner, I. Rish, M. Gupta, J. Moreira, S. Ma, R. Vilalta and A. Sivasubramaniam, "Critical Event Prediction for Proactive Management in Large-scale Computer Clusters," in Proc. SIGKDD'03, 2003, pp. 426-435.

[23] R. Wolski, N. Spring and J. Hayes, "The Network Weather Service: A Distributed Resource Performance Forecasting Service for Metacomputing," Fut. Gen. Comp. Systems, vol. 15, pp. 757-768, 1999.

[24] P. Dinda and D. O'Hallaron, "An Extensive Toolkit for Resource Prediction in Distributed Systems," School of Computer Science, Carnegie Mellon Univ., Technical Report CMU-CS-99-138, 1999.

[25] D. Anderson, "BOINC: A System for Public-Resource Computing and Storage," 5th IEEE/ACM Workshop on Grid Computing, Nov. 2004.

[26] (2007) Teragrid. http://www.teragrid.org

[27] (2007) Enabling Grids for EsciencE. http://public.eu-egee.org/

[28] (2007) Open Science Grid. http://www.opensciencegrid.org/

[29] B. Chun, D. Culler, T. Roscoe, A. Bavier, L. Peterson, M. Wawrzoniak and M. Bowman, "PlanetLab: an overlay testbed for broad-coverage services," ACM SIGCOMM Comp. Comm. Review, 33(3), July 2003.

[30] S. Androutsellis-Theotokis and D. Spinellis, "A Survey of Peer-to-Peer Content Distribution Tech.," ACMCS, 36(4), pp. 335-371, Dec. 2004.

[31] N. Abu-Ghazaleh and M. Lewis, "Toward Self Organizing Grids," in IEEE HPDC Hot Topics Session, 2006, p. 324-327.

[32] I. Foster and A. Iamnitchi, "On Death, Taxes, and the Convergence of Peer-to-Peer and Grid Computing," in Proc. IPTPS '03, Feb. 2003.

[33] J. Frey, T. Tannenbaum, M. Livny, I. Foster, and S. Tuecke, "CondorG: A Computation Management Agent for Multi-institutional Grids," in $H P D C^{\prime} 01,2001$, p. 55-63. 